\author{
Mohammad Reza Zareei \\ Mehdi Iranmanesh ${ }^{*}$
}

http://dx.doi.org/10.21278/brod69208

ISSN 0007-215X

eISSN 1845-5859

\title{
RELIABILITY-BASED INSPECTION PLANNING OF THE SHIP STRUCTURE EXPOSED TO FATIGUE DAMAGES
}

UDC 629.5.015.4:629.5.083

Original scientific paper

\begin{abstract}
Summary
Reliability-based inspection planning is one of the most popular methods in determining the time of inspection and repairs in various structures. In this way, inspection and repair times are determined mainly by putting a lower limit for the reliability index. The detection and measurement of cracks is one of the possible outputs at the time of inspecting fatigue cracking. One way to use this output is to update the parameters of the fatigue reliability equation. In this study, statistical distribution of the parameters of the problem is updated and fatigue reliability is calculated for inspection planning using the Bayesian updating concept through the Markov Chain Monte Carlo (MCMC) method and the Metropolis-Hasting algorithm. The distribution of crack growth equation material parameters and the initial crack length will be updated with this method. The application of the proposed method has been shown in a structural member of a ship.
\end{abstract}

Key words: $\quad$ Reliability-based; inspection planning; Fatigue failure; crack growth parameters; Bayesian updating; Markov Chain-Monte Carlo.

\section{Introduction}

Various structures such as marine structures are aging over time. Fatigue cracking is one of the main factors that can reduce the strength of the structural members of a ship with the passage of time, thus increasing the probability of failure. To maintain the safety of ship structures, some maintenance activities over the lifetime of ships should be conducted. These activities are carried out in the form of the inspection of different parts of the ship at certain times, and, if necessary, by repairing them.

For inspection planning of structures, various methods are provided, with reliabilitybased inspection planning being one of the most famous methods. In this way, the reliability index is calculated over the lifetime of the structure. The degree of structural reliability reduces over time. In this planning method, usually a lower limit for reliability is defined as the target reliability index to control the structural safety a ship. The first inspection is planned for the structure after reliability reaches the limit value. This process is schematically shown in Figure 1. 
No crack detection, crack detection without size measurement, and crack detection with size measurement are the three possible outputs of the inspection of the damage caused by fatigue cracking. Several studies (e.g. [1\&2]) have worked on the use of the results of inspections under fatigue failure. In these studies, mostly Bayesian theory and equations, including the equations proposed by Madsen in [3], have been used to update the failure probability and its corresponding reliability index. In these research studies, random variables of fatigue reliability equations after repairing the structural components are considered equal to the amounts prior to the repair. This means that the results of the inspection are not used to update the distribution of random variables of the problem. Accordingly, using the Bayesian approach, the reliability can be calculated after the first inspection and then the second inspection can be scheduled.

An alternative approach for the above-mentioned procedure is to update the random variables of the problem based on inspection results and then to calculate the structural reliability for planning the next inspection time. Little research has been carried out in this regard so far.

Garbatov and Guedes Soares [4] proposed a Bayesian approach to update some of the parameters of the probability distributions governing the reliability assessment of the maintained floating structures. Based on the time-dependent fatigue reliability and using the information from the inspections, the description of the time to crack initiation, crack growth law, and the probability of crack detection were updated. Heredia-Zavoni and MontesIturrizaga [5] investigated a Bayesian framework using an analytical model for updating the initial crack size distribution at a certain point in time for the tubular joints of fixed offshore structures. The crack size measurement is used for updating as the new data. Soliman and Frangopol [6] proposed a Bayesian updating approach to find the updated distributions of fatigue crack growth model parameters based on inspection results. The updated parameters were used to find the next inspection time based on a cost-based optimization approach.

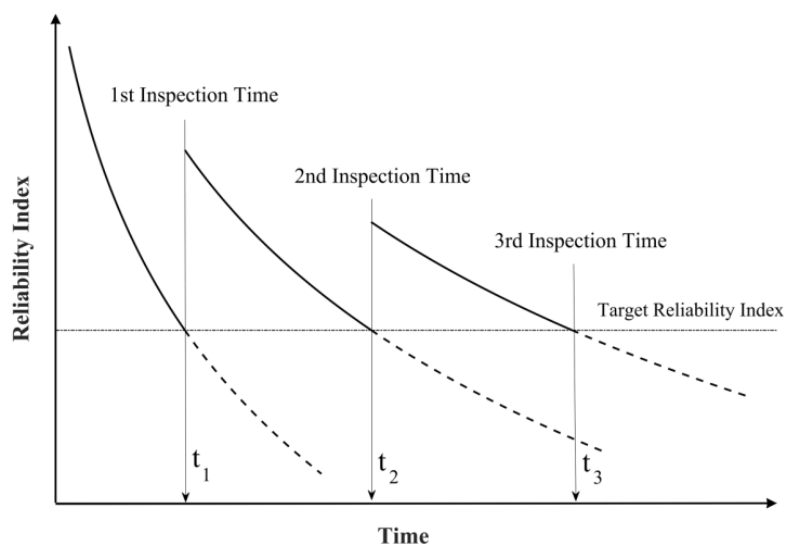

Fig. 1 Single figure example

In this study, the first structural inspection time is planned by calculating the fatigue reliability. After that, by assuming that cracks can be measured with different lengths at the time of the first inspection, the distribution of the parameters of the fatigue reliability equation has been updated with the Markov Chain Monte Carlo method by using the Bayesian updating concept. Finally, the next inspection time is planned after calculating the fatigue reliability using the updated parameters.

One of the features of the proposed approach is that it can be used in minimizing aging effect of ship structure integrity. The effect of fatigue cracks on aging effect of ship structures was previously investigated in some articles. By updating the distribution of effective 
parameters, a more accurate approximation of the parameters governing the problem can be studied and then the aging effects of structures can be investigated more accurately.

\section{Fatigue Failure}

According to linear elastic fracture mechanics, the Paris-Erdogan equation provides the crack growth as:

$$
\frac{d a}{d N}=\mathrm{C}(\Delta K)^{m}
$$

Where $a$ is the crack size, $N$ is the number of loading cycles, and $m$ and $C$ are material constants. $D K$ is the stress intensity range, which is given in the following:

$$
\Delta K=\Delta \sigma K(a) \sqrt{\pi a}
$$

Here $D s$ is the applied stress range and $K(a)$ is the geometry function. Integrating the above equation, the number of cycles of stress applying that is required for the crack growth from sizes $a_{1}$ to $a_{2}$ can be obtained from the following equation:

$$
N_{1-2}=\int_{a_{1}}^{a_{2}} \frac{d a}{C(\Delta \sigma K(a) \sqrt{\pi a})^{m}}
$$

Owing to random wave-induced loads, variable amplitude fatigue loading is inherent to ship structures, thus adding complexity to predicting the crack growth. Therefore, simplified models are preferred [7]. An equivalent constant amplitude stress range is a simplified method that renders the same degree of damage (i.e. crack growth) as the variable amplitude loading. It can be expressed as [8]:

$$
\Delta \sigma_{e q}=\left[\int_{0}^{\infty}(\Delta \sigma)^{\beta} f_{\Delta \sigma}(\Delta \sigma) d(\Delta \sigma)\right]^{1 / \beta}
$$

Where $\beta$ is the interaction coefficient and $f_{\Delta \sigma}(\Delta \sigma)$ is the probability density function of the stress range.

By integrating Equation 1 and using an equivalent stress range, the following safety margin can be used for calculating the fatigue reliability as:

$$
G=\int_{a_{0}}^{a_{c}} \frac{d a}{(K(a) \sqrt{\pi a})^{m}}-\Delta \sigma_{e q}{ }^{m} C N
$$

Where $a_{0}$ and $a_{c}$ are initial and critical crack lengths. The probability of failure $P_{f}$ and the reliability index $\beta$ can be obtained as:

$$
P_{f}=P[G<0]=\Phi(-\beta)
$$

Where $F()$ is the standard normal distribution function.

Reliability evaluation can be performed with approximate methods like First Order Reliability Method (FORM), Second Order Reliability Method (SORM) or Monte Carlo simulation (MCS). In the present study, the probability of failure and the corresponding reliability indices are calculated by the MCS method. 
The Weibull distribution is an adequate approximation for the long-term stress range in ship structural members. Hence, the m-th statistical moment of the long-term stress range can be expressed in terms of Scale $A$ and shape parameters $B$ of the Weibull distribution as:

$$
\Delta \sigma_{e q}^{m}=A^{m} \Gamma\left(1+\frac{m}{B}\right)
$$

In this study, with Bayesian updating, it has been tried to obtain the updated distribution of the model parameters, including $a_{0}, m$, and $C$, using new data of the crack size measured in the inspection time. By updating these parameters, Equation 5 is rewritten as:

$$
G_{u p d}=\int_{a_{0_{u p d}}}^{a_{c}} \frac{d a}{(K(a) \sqrt{\pi a})^{m_{u p d}}}-\Delta \sigma_{e q}^{m_{u p d}} C_{u p d} N
$$

Where $a_{0_{u p d}}$ is the updated initial crack length, and $m_{\text {upd }}$ and $C_{\text {upd }}$ are the updated material constants.

\section{Bayesian theorem}

The Bayesian theory has been widely used to update the parameters of various equations. This theory can be rewritten to update the required parameters of the reliability equation. Based on this, the updated or posterior distribution of the parameters can be obtained by combining the prior distribution of the parameters with new information on the crack size obtained from the structural inspection. The Bayesian theory can be rewritten based on probability distribution functions (PDFs). Hence, in this regard, $R$ and $S$ represent the model parameter and measured parameter, respectively [9]:

$$
f_{R}(r \mid S=s)=\frac{f_{S}(r \mid R=r) f_{R}(\mathrm{r})}{f_{S}(s)}
$$

Where $f_{R}(r)$ is the prior PDF of the model parameters, $f_{S}(s)$ is the PDF of the measurement, $f_{S}(s \mid R=r)$ is the likelihood function of obtaining the measured values conditioned on the estimated model parameters, and $f_{R}(r \mid S=s)$ is the updated or posterior PDF of the model parameters. The denominator in the above equation is the normalization constant [10] and thus it can be removed from the equation as:

$$
f_{R}(r \mid S=s) \propto f_{R}(\mathrm{r}) f_{S}(r \mid R=r)
$$

\subsection{Bayesian updating}

It is assumed that a member has been subjected to fatigue failure and the Paris-Erdogan equation governs the growth of cracks. The general model for crack growth prediction can be expressed as:

$$
a_{d}=M(N, x ; y)+e
$$

Where $a_{d}$ is the measured value of the crack size and $e$ is the general error term that includes the measurement error $t$ and the modeling error e. $M(N, x ; y)$ is the crack growth model in which $x$ is the model parameter for updating and $y$ is the model independent variable. $N$ in the above equation is a non-random variable such as the number of applied stress cycles or time. 
Model parameters in the problem include $a_{0}, m$, and $\ln (C)$ (natural logarithm of the material parameter $C$ ) that create the model parameter vector $x=\left\{\ln (C), m, a_{0}\right\}$. The error terms $e$ and $t$ are usually considered as normal variables with zero mean and finite variances of $\mathrm{se}^{2}$ and $\mathrm{St}^{2}$. The likelihood function has been defined as [11]:

$$
L\left(a_{d} \mid x\right)=\frac{1}{\sqrt{2 \pi} \sqrt{\sigma_{\varepsilon}{ }^{2}+\sigma_{\tau}^{2}}} \exp \left(-\frac{1}{2} \frac{\left(a_{d}-M(N, x ; y)\right)^{2}}{\left(\sigma_{\varepsilon}{ }^{2}+\sigma_{\tau}{ }^{2}\right)}\right)
$$

The general error term $e \sim \operatorname{Normal}\left(0, s_{e}{ }^{2}\right)$ can be substituted instead of measurement and modelling errors, and, then, the likelihood function can be defined as:

$$
L\left(a_{d} \mid x\right)=\frac{1}{\sqrt{2 \pi} \sigma_{e}} \exp \left(-\frac{1}{2} \frac{\left(a_{d}-M(N, x ; y)\right)^{2}}{\sigma_{e}^{2}}\right)
$$

This function represents the probability of detecting a crack with size ad after the $N$ loading cycles provided the variable input vector $x$. If the crack size is measured " $n$ " times in different cycles for a particular component, the likelihood function can be defined as:

$$
L\left(a_{d, 1}, a_{d, 2}, \ldots, a_{d, \mathrm{n}} \mid x\right)=\frac{1}{\left(\sqrt{2 \pi} \sigma_{e}\right)^{n}} \exp \left(\sum_{i=1}^{n}-\frac{1}{2} \frac{\left(a_{d, i}-M\left(N_{i}, x\right)\right)^{2}}{\sigma_{e}{ }^{2}}\right)
$$

Here $a_{d, i}$ are measured values of crack sizes at $N_{i}$ corresponding loading cycles. Putting the likelihood function in Equation 10, it is possible to calculate the posterior or updated distribution of model parameters.

\subsection{Markov Chain Monte Carlo (MCMC)}

It is difficult to find an analytical solution of Equation 10 in general terms and that is why simulation-based methods, such as the MCMC method, are used. Here the cascade Metropolis-Hasting algorithm has been used. The Markov chain is formed in this method by iterative sampling. The convergence of the chain is among the features of the Markov chain regardless of the starting point. To update the parameter $\mathrm{x}$, it starts from an initial value $\mathrm{x}_{1}$ in a chain and the value $x_{i+1}$ is produced in a way that is independent of $x_{1}, \ldots, x_{i-1}, x_{i}$. This process is as the following [9]:

1. Each chain starts at $i=1, x_{1}$.

2. Generate a random value $x^{*}$ using the transition or proposal density function, $q(x \mid x i)$

The proposal distribution function $q$ is usually zero mean normal or uniform density function; therefore, it is a symmetric function.

$$
q\left(x^{*} \mid x_{i}\right)=q\left(x^{*}-x_{i}\right)=q\left(x_{i}-x^{*}\right)
$$

3. Prior acceptance of the probability evaluation is given as the following:

$$
\alpha_{p}=\min \left\{1, \frac{f_{x}\left(\mathrm{x}^{*}\right)}{f_{x}\left(\mathrm{x}_{i}\right)} \frac{q\left(\mathrm{x}_{i} \mid \mathrm{x}^{*}\right)}{q\left(\mathrm{x}^{*} \mid \mathrm{x}_{i}\right)}\right\}
$$

Considering the symmetry of proposal density function: 


$$
\alpha_{p}=\min \left\{1, \frac{f_{x}\left(\mathrm{x}^{*}\right)}{f_{x}\left(\mathrm{x}_{i}\right)}\right\}
$$

Where $f_{x}(x)$ is the prior PDF of input parameters.

4. Calculation of $u_{p} \sim$ Uniform $[0,1]$

- If $u_{p}>\alpha_{p}\left(x_{i}, x^{*}\right)$, accept and go to Step 5 .

- Otherwise, go to Step 2.

5. The likelihood acceptance of probability evaluation is given as:

$$
\alpha_{L}=\min \left\{1, \frac{L_{x}\left(\mathrm{x}^{*}\right)}{L_{x}\left(\mathrm{x}_{i}\right)}\right\}
$$

Where $L_{x}$ is the likelihood given in Equations 13 and 14 .

6. Calculation of $u_{L} \sim$ Uniform $[0,1]$

- If $u_{L}>\alpha_{L}\left(x_{i}, x^{*}\right)$ accept and set $x_{i+1}=x^{*}, i+1=i$ and go to Step 2 .

- Otherwise go to Step 2.

This process should be repeated to achieve the desired number of elements in the Markov chain. To make the chain independent of its initial value, the initial portion of the chain, which is known as the burn-in portion, will be discarded.

The convergence of the chain is the important issue in using this method. Various methods have been proposed to evaluate the convergence of the Markov chain. Here the approach provided by [12] is used. According to this method, multiple chains should be created with different starting points. Variances within each chain are compared with the variances between chains [9]. The large difference between these two values indicates a lack of convergence. If there are $\mathrm{v}$ chains with $\mathrm{n}$ elements (which burn-in period is discarded), the variance between chains, $\mathrm{B}$, is obtained as:

$$
\frac{B}{n}=\frac{1}{v-1} \sum_{j=1}^{v}\left(\overline{X_{j}}-\bar{X}\right)^{2}
$$

In the above equation, $\overline{X_{j}}$ is the average value of the $j$-th chain, and $\bar{X}$ is the average value of all chains. The within chain variance is calculated as:

$$
W=\frac{1}{v(n-1)} \sum_{j=1}^{v} \sum_{i=1}^{n}\left(X_{j i}-\overline{X_{j}}\right)^{2}
$$

Where $X_{j i}$ is the $i$-th element of the $j$-th chain. The variance ratio $\mathrm{R}$ is defined as:

$$
\begin{aligned}
& R=\frac{\overline{\bar{V}}}{W} \\
& \overline{\bar{V}}=\frac{n-1}{n} W+\frac{v+1}{v n} B
\end{aligned}
$$

Accordingly, if chains converge to the target distribution, $R$ should be close to 1 . According to [12], the $\mathrm{R}$ value less than 1.1 or 1.2 represents the convergence of the method. Here we consider the amount of 1.2 as the convergence criteria. Initial samples with the variance ratio greater than 1.2 will be discarded, while the other samples will be used to describe the posterior or updated distribution function. 


\section{Verification example}

Virkler et al. [13] reported the results of a set of crack growth experiments on the Aluminium 2024 alloy. A total of 68 centre-through crack specimens with the same loading, geometry, and material were tested. The specimens were tested with the initial length of crack $a_{0}=9 \mathrm{~mm}$, the width $w=154.2 \mathrm{~mm}$, the thickness $d=2.54 \mathrm{~mm}$, and under a constant tension of $48.82 \mathrm{MPa}$. The geometry function for Virkler samples is as:

$$
K\left(\frac{a}{w}\right)=\sqrt{\sec \left(\frac{\pi a}{w}\right)}
$$

Several researchers have analysed the results of these tests. In [11], a total of 15 crack growth curves (Figure 2) were selected for achieving the prior distribution of $\{\ln (C), m\}$. Also, according to the correlation between these two variables, a bivariate normal distribution was proposed as:

$$
\begin{aligned}
& p_{0}(\ln C, m)=\frac{1}{2 \pi \sqrt{\Sigma}} \exp \left(-\frac{1}{2}\left[\begin{array}{c}
\ln C+26.7060 \\
m-2.9684
\end{array}\right]^{\prime} \times \Sigma^{\prime} \times\left[\begin{array}{c}
\ln C+26.7060 \\
m-2.9684
\end{array}\right]\right) \\
& \left.\Sigma=\left[\begin{array}{cc}
0.5335 & -0.0886 \\
-0.0886 & 0.0148
\end{array}\right]\right)
\end{aligned}
$$

$\Sigma$ is the covariance matrix of these two variables. According to the prior distribution of the material parameters, the crack growth curve is shown in Figure 3. On this curve, the average and the range of $95 \%$ confidence interval (CI) have been shown.

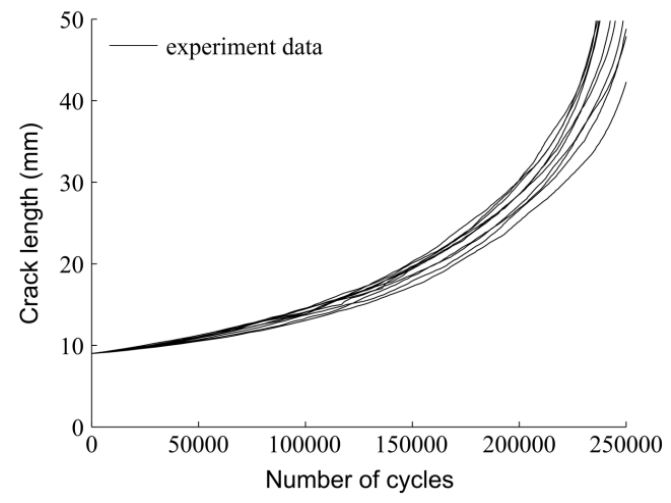

Fig. 2 Experimental data for parameter identification of Virkler tests [11]

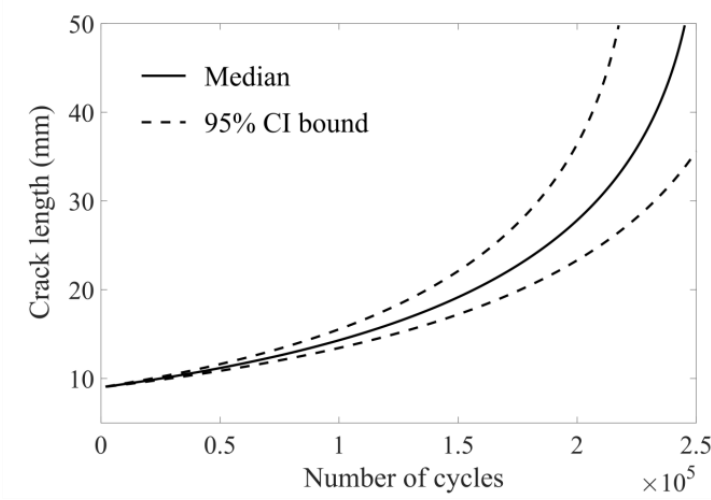

Fig. 3 Crack growth curve using prior distribution of parameters

To update the parameters, additional data on the crack size is required. This additional data can be obtained through inspection or health monitoring. Here three points of a crack growth curves provided by [11] are used as measured crack sizes in different cycles for updating. This is shown in Table 1. In the three steps, each paired data of Table 1 has been used to perform the update. As many as four chains with 30,000 elements are formed to update the parameters. Here $s_{e}=0.2 \mathrm{~mm}$ is considered. After updating, the distribution of parameters with respect to each of the points 1 to 3 is shown in Figure 4. The mean vector and the covariance matrix associated with prior and posterior distributions are obtained in Table 2.

Crack growth curves using posterior distributions are shown in Figure 5. Comparing Figures 3 and 5, it can be seen that using additional data on the crack size, the average value, as shown in Figure 5, is inclined towards the additional crack size information based on the 
data given in Table 1, while the range of CI of $95 \%$ decreases. These two effects show the reduced uncertainty of crack growth parameters.

Table 1 Additional information of crack growth size for model parameter updating [11]

\begin{tabular}{lll}
\hline Point & Cycles number $N$ & Crack size $a(\mathrm{~mm})$ \\
\hline 1 & 38485 & 10 \\
2 & 64064 & 11 \\
3 & 85164 & 12 \\
\hline
\end{tabular}

Table 2 Prior and posterior distributions of crack growth parameters

\begin{tabular}{llc}
\hline Point used & Mean vector $\{\ln (C), m\}$ & \multicolumn{2}{c}{ covariance matrix } \\
\hline Prior & $\{-26.7060,2.9684\}$ & {$\left[\begin{array}{cc}0.5335 & -0.0886 \\
-0.0886 & 0.0148\end{array}\right]$} \\
1 & $\{-27.4128,3.0767\}$ & {$\left[\begin{array}{cc}0.4637 & -0.0782 \\
-0.0782 & 0.0133\end{array}\right]$} \\
2 & $\{-27.8356,3.1404\}$ & {$\left[\begin{array}{cc}0.4003 & -0.00684 \\
-0.0684 & 0.0117\end{array}\right]$} \\
3 & $\{-28.0052,3.1656\}$ & {$\left[\begin{array}{cc}0.3477 & -0.0600 \\
-0.0600 & 0.0104\end{array}\right]$} \\
\hline
\end{tabular}

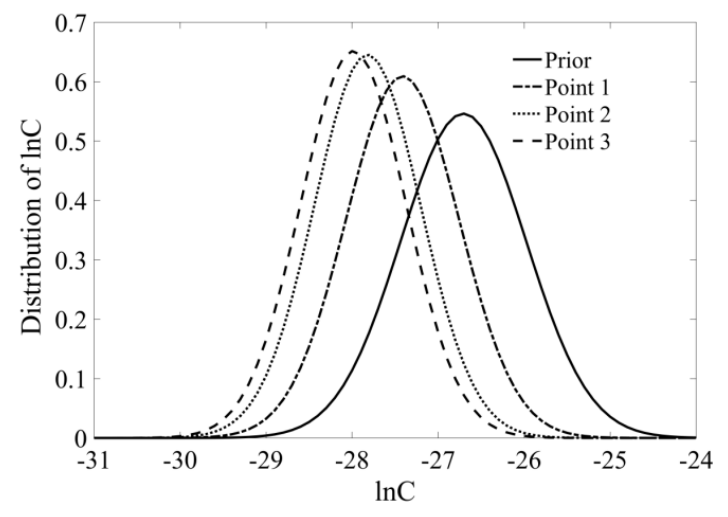

(a)

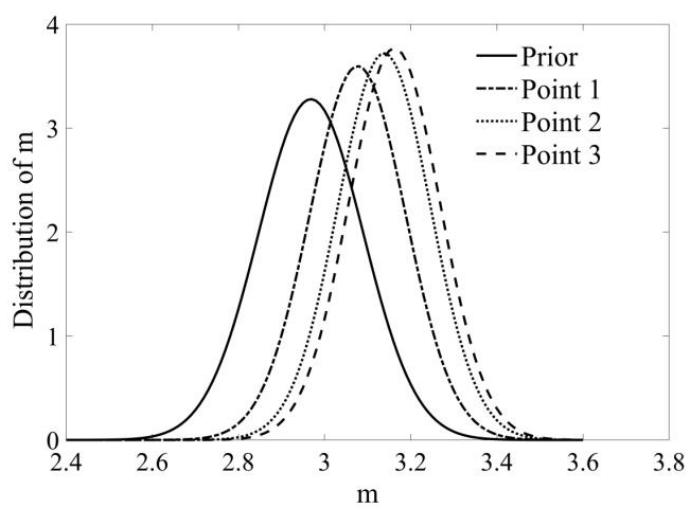

(b)

Fig. 4 Posterior distribution of parameters using crack sizes: (a) $\ln (c)$, (b) $m$

In this example, the critical crack length is considered equal to $49.8 \mathrm{~mm}$. Considering the constant loading stress and using Equation 5, the failure probability curves are provided based on prior and posterior distributions in Figure 6. As is evident from this figure, the use of updated parameters based on the additional data on crack size has a very significant effect on failure probability values in various cycles.

\section{Application example}

To illustrate the application of model parameter updating in reliability-based inspection planning, a ship structural detail at the intersection between deck transverse and deck longitudinal is considered. This detail is selected from an article of [7] and shown in Figure 7. The critical crack depth is assumed to be the flange thickness. It is assumed that this detail is located in deck structure of a tanker with $237 \mathrm{~m}$ length.

The geometry function for a semi-elliptical crack shape is defined as [7]:

$$
K(a)=K_{E} K_{S} K_{T} K_{G}
$$




$$
K_{E}=\text { basic crack shape factor }=\left[1+4.5945 \cdot\left(\frac{a}{2 c}\right)^{1.65}\right]^{0.5}
$$

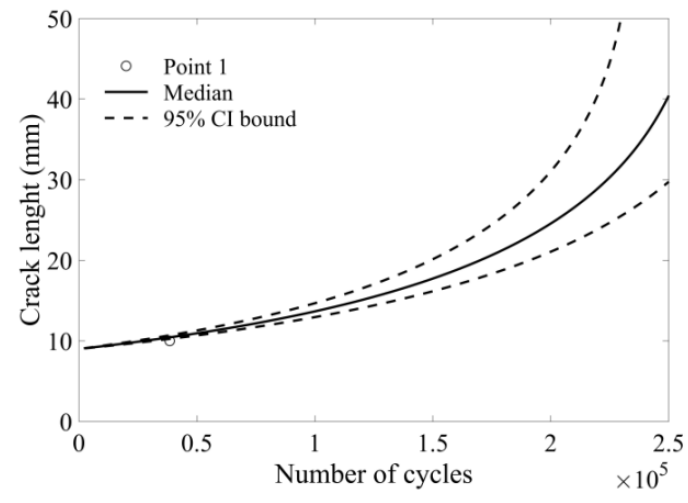

(a)

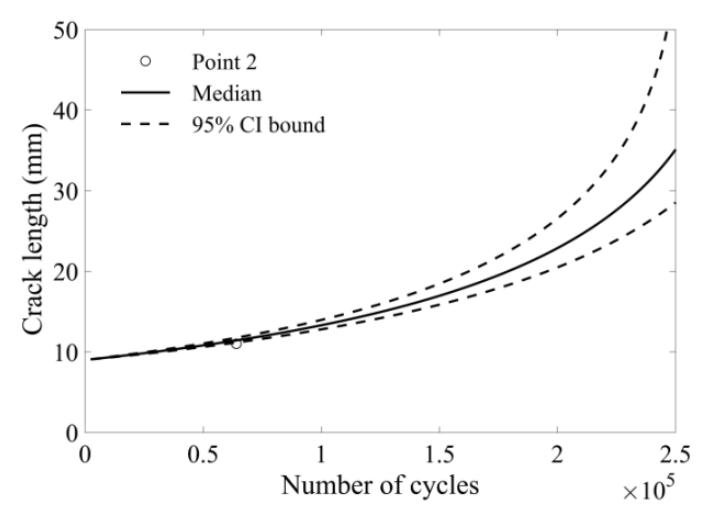

(b)

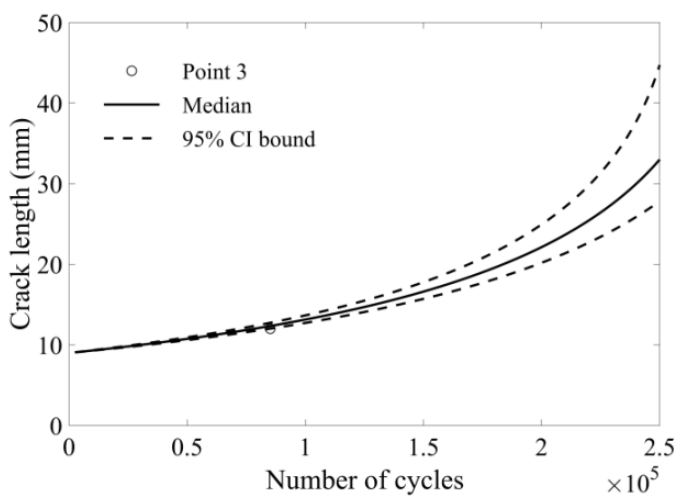

(c)

Fig. 5 Crack growth curve using posterior distribution of parameters related to: (a) Point 1, (b) Point 2, (c) Point 3.

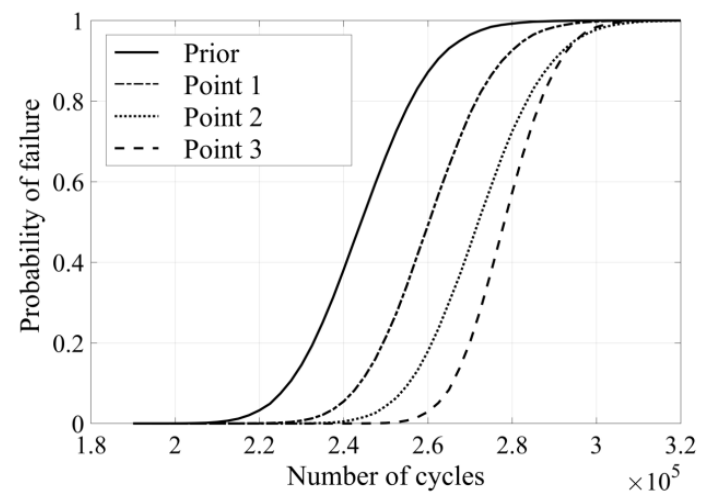

Fig. 6 Failure probability curves using prior and posterior distributions

$$
\begin{aligned}
& K_{S}=\text { front face factor }=1-0.16 \cdot\left(\frac{a}{2 c}\right) \\
& K_{T}(x=a / 2 c ; y=a / h)=\text { finite thickness factor }=1+\left(0.008 y x^{-2.454}+0.0534 y^{2} x^{-1.005}\right)
\end{aligned}
$$




$$
K_{G}=\text { Stress gradient factor }=\frac{S C F}{1+\frac{(a / h)^{0.6051}}{1.158}}
$$

Here $S C F$ is the stress concentration factor, which is considered to be equal to 2.1. Also, the crack aspect ratio is assumed to be $2 c=6.71+2.58 a$.

All necessary data for predicting crack size and calculating reliability index is provided in Table 3. The initial crack size is assumed to follow a normal distribution with mean value of 0.5 $\mathrm{mm}$ and standard deviation of $0.05 \mathrm{~mm}$ [6]. The natural logarithm of the material parameter $C$ $(\ln (C))$ is treated as normal distribution random variable with a mean value of -29.97 and standard deviation of 0.514 [14] while the material parameter $\mathrm{m}$ is assumed to follow a normal distribution with a mean value of 3.0 and standard deviation of 0.15 . The correlation coefficient between $\mathrm{m}$ and $\ln (C)$ is assumed to be -0.9 [15].

It is assumed that the long-term stress range of the detail following the Weibull distribution. Parameters of stress range can be calculated from DNV [16]. For a detail located in deck structure, the Weibull shape parameter can be calculated as:

$$
B=2.21-0.54 \log _{10}(L)
$$

The Weibull scale parameter $A$ can be defined as:

$$
A=\frac{\Delta \sigma_{0}}{\left(\ln n_{0}\right)^{1 / h}}
$$

Here, $\Delta \sigma_{0}$ is the reference stress range value at the local detail, which exceeded once out of no cycles, and $n_{0}$ is the total number of cycles associated with the stress range level $\Delta \sigma_{0}$. In the present study, it is assumed that $\Delta \sigma_{0}$ is equal to $125 \mathrm{MPa}$. Shape and scale parameters are respectively obtained as 0.9276 and 13.288 . The number of cycles is defined as $N=n_{0 . t}$ that $\mathrm{t}$ is the time and $n_{0}$ is the long-term average zero crossing frequency. The frequency $n_{0}$ can be obtained as [16]:

$$
v_{0}=\frac{1}{4 \cdot \log _{10}(L)}
$$

The frequency $n_{0}$ is calculated as $0.1053 \mathrm{~Hz}$ and hence the annual number of cycles is $3.32 \mathrm{e} 6$.
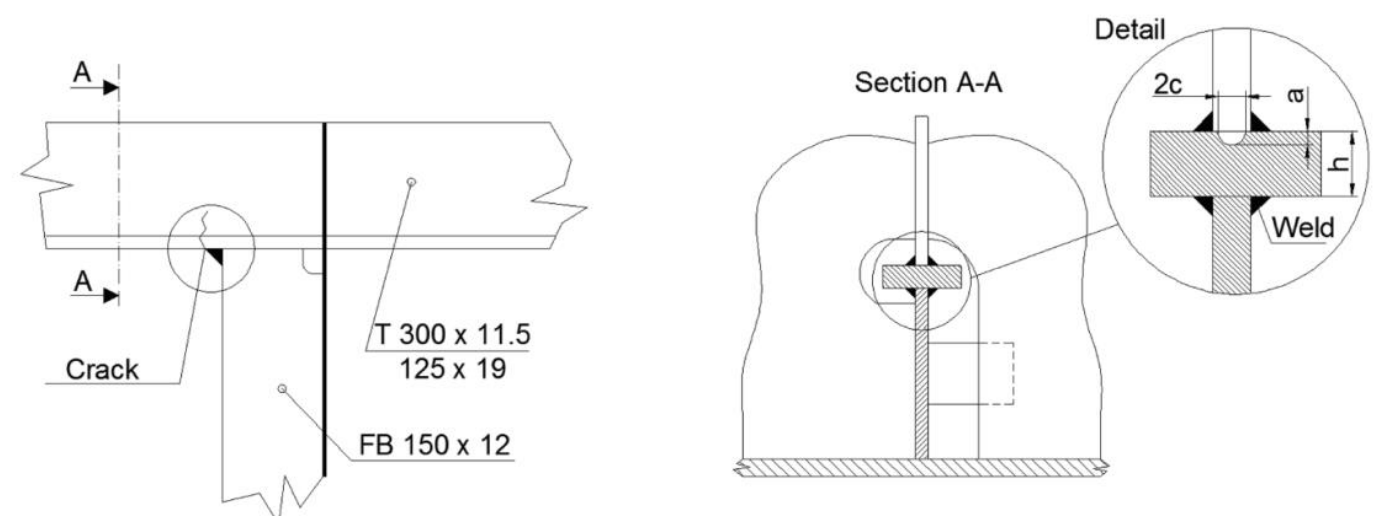

Fig. 7 Ship structural detail and crack location [7] 


\subsection{Estimation of first inspection time}

The reliability index over time is calculated by Equation 5 and shown in Figure 8. The first inspection is done at a time when the reliability index is equal to the target reliability index. Several studies have focused on the establishment of the target reliability index. Mansour [17] proposed values for the target reliability index in accordance with Table 4.

Table 3 Variable for crack growth prediction and reliability calculation

\begin{tabular}{ccccc}
\hline Variable & $\begin{array}{c}\text { Notation } \\
\text { (units) }\end{array}$ & Mean & Standard deviation & Distribution type \\
\hline Initial crack size & $a_{0}(\mathrm{~mm})$ & 0.5 & 0.05 & Normal \\
Material parameter & $\operatorname{Ln}(C)$ & -29.97 & 0.514 & Normal \\
Annual number of & $m$ & 3.0 & 0.15 & Normal \\
cycles & $N_{a n}$ & $3.32 \times 10^{6}$ & - & Deterministic \\
\hline
\end{tabular}

The first inspection times for different values of the target reliability index are given in Table 5. Also, the mean values of crack size at the first inspection time are presented in Table 5. In this study, the point 2.5 has been considered as the target reliability index. Based on prior distributions of the parameters, the structure must be inspected in 6.72 years and the mean value of crack size at this time is $2.07 \mathrm{~mm}$.

Table 4: Target reliability indices [17]

\begin{tabular}{ccc}
\hline Consequences & Tanker & Cruiser \\
\hline Not serious & 2.0 & 2.5 \\
Serious & 2.5 & 3.0 \\
Very serious & 3.0 & 3.5 \\
\hline
\end{tabular}

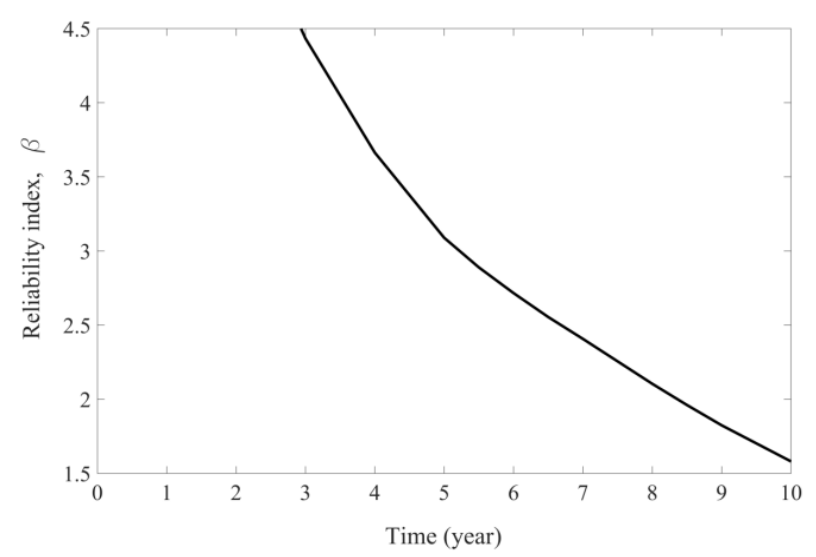

Fig. 8 Fatigue reliability index of the selected structural detail

Table 5: First inspection times

\begin{tabular}{ccc}
\hline Target reliability index & First inspection time (year) & Mean crack size $(\mathrm{mm})$ \\
\hline 3.0 & 5.43 & 1.62 \\
2.5 & 6.72 & 2.07 \\
2.0 & 8.4 & 2.78 \\
\hline
\end{tabular}




\subsection{Model parameter updating and next inspection time}

As noted earlier, the parameters $\ln (C), m$, and $a_{0}$ are updated with the MCMC method using the Metropolis-Hasting algorithm in this section. Prior distributions of these variables are presented in Table 3. Two Markov chains with 30,000 elements are considered. To investigate the convergence, the variances ratio is considered equal to $1.2 . s_{e}=0.2 \mathrm{~mm}$ is considered.

Additional information of crack size is required for parameter updating. Here it is assumed that cracks of various sizes have been detected at the first inspection time and thus the effect of the measurement of the cracks with different sizes on the posterior distribution of the parameters is investigated. It is assumed that cracks with lengths equal to $1,1.5,2.5,3$, and 4 $\mathrm{mm}$ during the first inspection time are detected.

A sample curve of the variance ratio associated with the detection of a crack with the length of $1.0 \mathrm{~mm}$ at the first inspection time is shown in Figure 9. Based on this figure, the initial 10,000 elements are discarded from the chain, while the others are used to describe the posterior distribution. Prior and posterior distributions of the parameters are shown in Figure 10. The mean values and standard deviations of the posterior distributions based on different crack size measurement values are shown in Table 6. In general, it can be said that with updating, the standard deviation of parameters has decreased, which implies the reduced uncertainty in the parameters. Any crack detected with a length lower than the mean value of $2.07 \mathrm{~mm}$ increases the average value of $\ln (C)$ and decreases the average values $\mathrm{a}_{0}$ and $\mathrm{m}$ and vice versa. The larger crack is detected at the first inspection time, where the lower standard deviation of $\ln (C)$ and $m$ are observed. Also, the correlation coefficient between the two variables is also reduced. A similar process can be observed for verification.

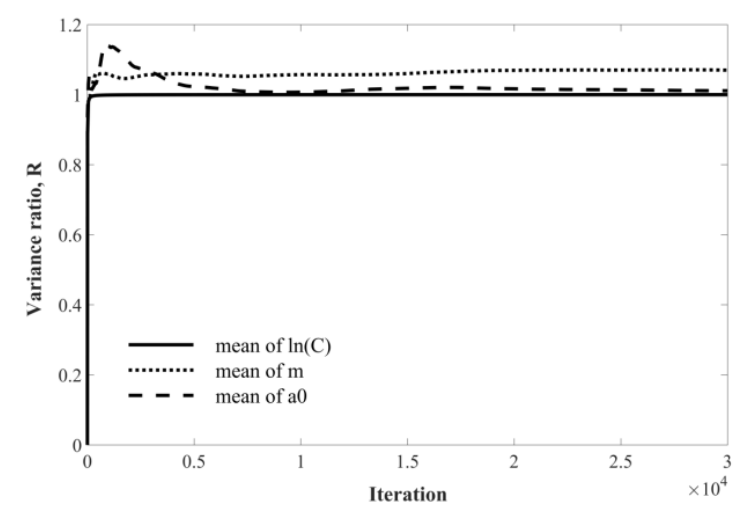

(a)

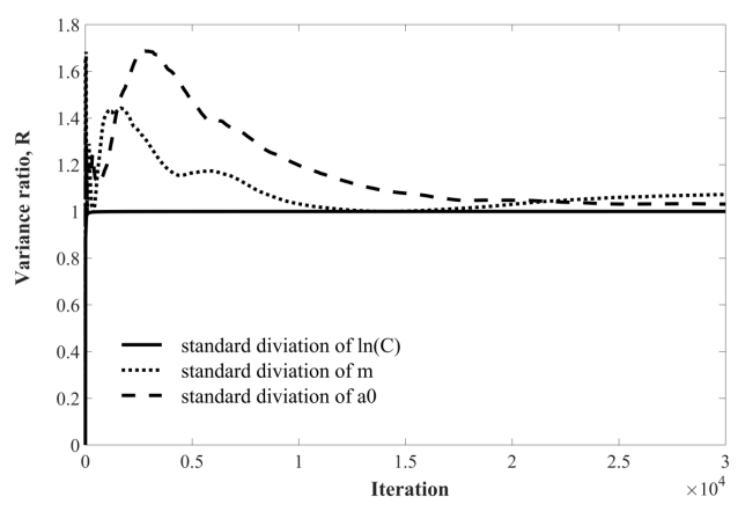

(b)

Fig. 9 Variance ratio in the Markov Chain Monte Carlo iterations: (a) R for mean values, (b) R for standard deviation values

The curve of mean crack size has been shown in Figure 11. Based on this figure, if a crack larger than $2.07 \mathrm{~mm}$ is detected, the slope of the crack growth curve increases and vice versa. The mean crack size at the time equal to first inspection time, 6.72 years, has been shown in the last column of Table 7 considering the updated parameters. As can be seen, considering the updated values of parameters, the mean value of crack size is very close to the assumed measured values of the cracks. For instance, when it is assumed that a crack with $3.0 \mathrm{~mm}$ is detected in the first inspection time and updating the parameters, the mean value of crack size at 6.72 years will be $2.95 \mathrm{~mm}$. This shows that posterior distributions can predict the crack size with more accuracy.

After updating the parameters of the problem, fatigue reliability is calculated by using Equation 8 and shown in Figure 12. Assuming a target reliability index value of 2.5, the time interval between the first and second inspections is presented in Table 7. 
Table 6: Prior and posterior distributions of crack growth parameters

\begin{tabular}{cccccccc}
\hline $\begin{array}{c}\text { Crack size } \\
\text { measured } \\
(\mathrm{mm})\end{array}$ & \multicolumn{3}{c}{ Mean Value } & \multicolumn{3}{c}{ Standard deviation } & Correlation \\
\hline Prior & -29.97 & 3.00 & 0.500 & 0.5140 & 0.1500 & 0.0500 & -0.900 \\
1.0 & -29.57 & 2.79 & 0.479 & 0.4740 & 0.1077 & 0.0489 & -0.943 \\
1.5 & -29.74 & 2.88 & 0.498 & 0.4736 & 0.1010 & 0.0504 & -0.962 \\
2.5 & -29.98 & 3.01 & 0.502 & 0.4580 & 0.0899 & 0.0505 & -0.986 \\
3.0 & -30.08 & 3.06 & 0.503 & 0.4578 & 0.0881 & 0.0496 & -0.991 \\
4.0 & -30.20 & 3.11 & 0.508 & 0.4383 & 0.0829 & 0.0524 & -0.993 \\
\hline
\end{tabular}

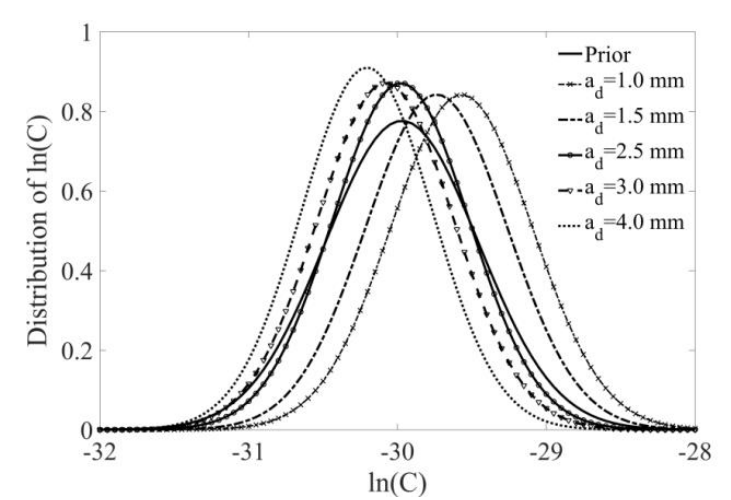

(a)

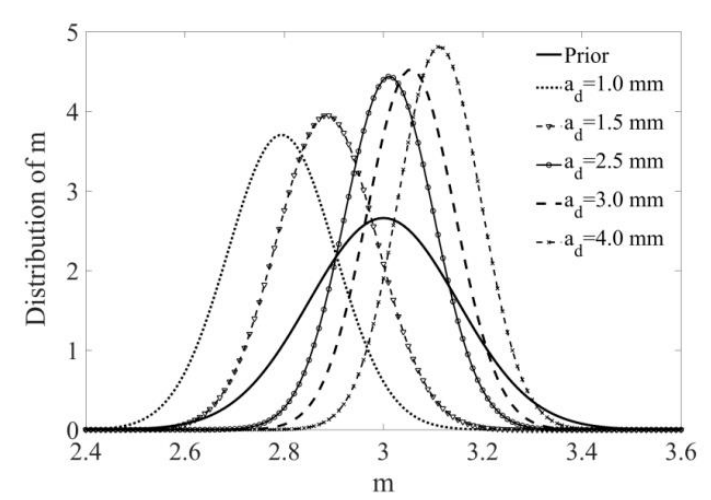

(b)

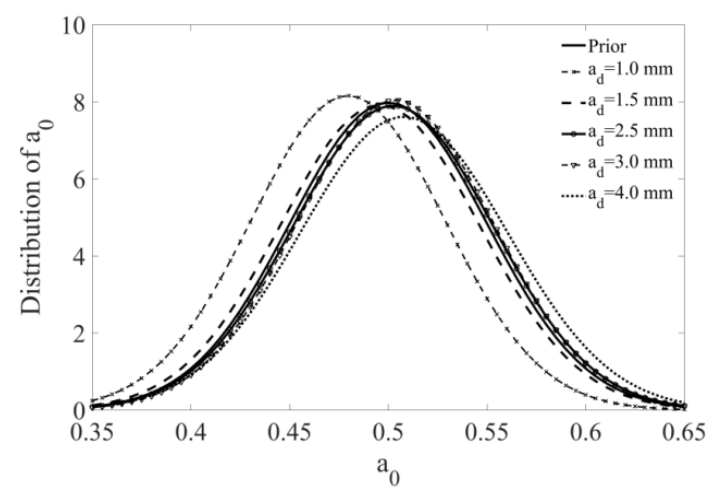

(c)

Fig. 10 Posterior distribution of parameters using inspection measurements: (a) $\ln (c)$, (b) $m$, (c) $a_{0}$

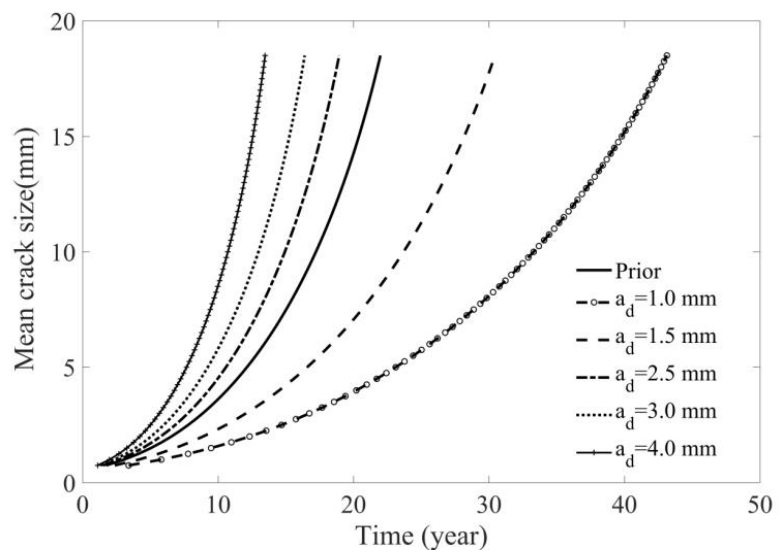

Fig. 11 Curve of mean crack size using posterior distribution of parameters based on inspection measurements 
From these results, it can be said that the larger the crack size measured at the time of inspection, the earlier is the re-inspection of the structure. Also, the second inspection time of the structure can be predicted with more accuracy by reducing the uncertainty of the parameters. Furthermore, due to the reduction in uncertainty in model parameters, by measuring the larger crack size the updated reliability curve decreases more rapidly. As expected, the size of the detected crack has a significant impact on the estimation of the second inspection time.

Table 7: Time interval between second and first inspection and mean crack size at time of first inspection

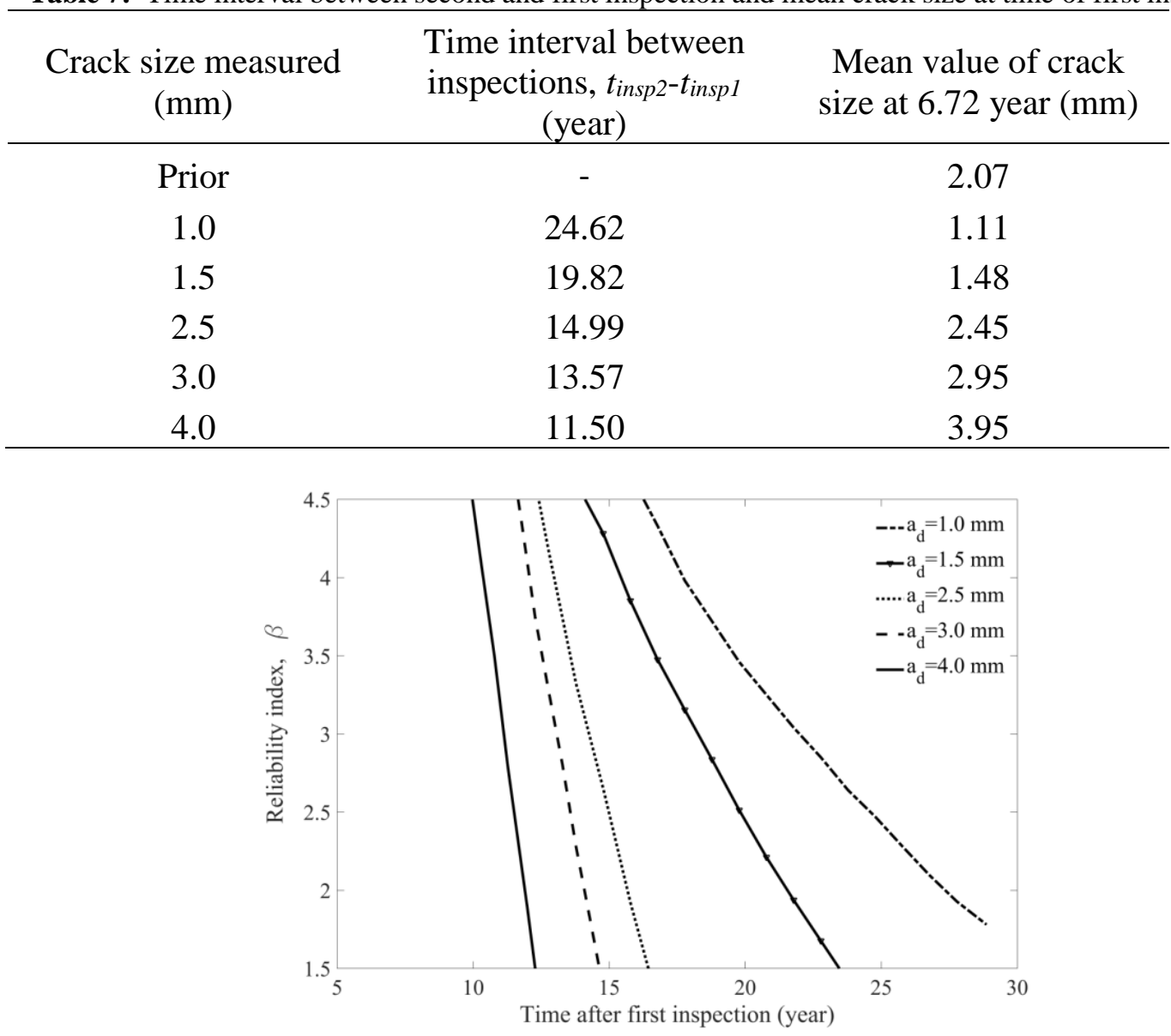

Fig. 12 Fatigue reliability index based on time after first inspection

\section{Conclusion}

Fatigue is a major cause of failure in steel structures. Fatigue reliability assessment is one of the methods for scheduling the inspection times of a structure. The prior distributions of parameters in fatigue reliability formulae can be achieved by performing various experiments or through the existing rules. In conventional methods, these distributions are also used for evaluating the reliability after the repair. One of the outputs of the structural inspection is the detection and measurement of crack length. Here the results of measuring the crack length at the time of inspection are used to update the distribution of the parameters of fatigue reliability formula. For this, the Bayesian updating concept along with the Markov Chain Monte Carlo (MCMC) method with the Metropolis-Hastings algorithm has been used. In this paper, the proposed method is used to update the material parameters in the equation of crack growth $(\ln (C)$ and $m$ in the Paris-Erdogan equation) and initial crack length. From the results, the following items can be concluded: 
1. Any update of the parameters reduces their uncertainty and thus the behaviour of the growth of cracks in the structure can be predicted with more certainty.

2. If during the inspection time of the structure there is a possibility to measure the crack length with high accuracy, then the structure lifetime and future inspection times can be estimated with higher accuracy.

3. The proposed approach can be used for other deteriorating mechanisms of ship structures such as corrosion.

4. During the inspection time of the structure, if cracks with much higher or lower than the predicted size are detected, they must be re-examined along with other conditions relating to the problem such as loading. In general terms, this method can also be used in the update of loading conditions.

\section{REFERENCES}

[1] Onoufriou, T., (1999). Reliability based inspection planning of offshore, J. Marine Structures, 12: 521539. https://doi.org/10.1016/S0951-8339(99)00030-1.

[2] Chen, N.Z., Wang, G., Guedes Soares, C., (2011). Palmgren-Miner's rule and fracture mechanics-based inspection planning, J. Engineering Fracture Mechanics 78: 3166-3182. https://doi.org/10.1016/j.engfracmech.2011.08.002.

[3] Madsen, H.O., (1997). Stochastic modeling of fatigue crack growth and inspection. In: Guedes Soares C. editor. Probabilistic Methods for Structural Design. Kluwer, Dordrecht, Netherlands, 59-84. https://doi.org/10.1007/978-94-011-5614-1_4.

[4] Garbatov, Y., Guedes Soares, C., (2002). Bayesian Updating in the Reliability Assessment of Maintained Floating Structures. J. Offshore Mechanics and Arctic Engineering 124(3): 139-145. https://doi.org/10.1115/1.1493200.

[5] Heredia-Zavoni, E., Montes-Iturrizaga, R., (2004). A Bayesian Model for the Probability Distribution of Fatigue Damage in Tubular Joints. J. Offshore Mechanics and Arctic Engineering 126(3): 243-249. https://doi.org/10.1115/1.1782645.

[6] Soliman, S.M., Frangopol, D.M., (2014). Life-Cycle Management of Fatigue-Sensitive Structures Integrating Inspection Information. J. Infrastructure Systems 20(2): 04014001. https://doi.org/10.1061/(ASCE)IS.1943-555X.0000169.

[7] Parunov, J., Gledić, I., Garbatov, Y., Guedes Soares, C., (2013). Fatigue assessment of corroded deck longitudinals of tankers. International Journal of Maritime Engineering 155(PART A): A9-A21.

[8] Hughes, O.F., Paik, J.K., (2010). Ship structural analysis and design. Jersey City (NJ), Society of Naval Architect and Marine Engineers.

[9] Rastogi, R., Ghosh, S., Ghosh, A.K., Vaze, K.K., Singh, P.K., 2016. Fatigue crack growth prediction in nuclear piping using Markov chain Monte Carlo simulation, J. Fatigue \& Fracture of Engineering Materials \& Structures 40(1): 145-156. https://doi.org/10.1111/ffe.12486.

[10] Martinez, W.L., Martinez, A.R., (2002). Computational statistics handbook with MATLAB. Chapman \& Hall/CRC, Boca Raton.

[11] Guan, X., Giffin, A., Jha, R., Liu, Y., (2012). Maximum relative entropy-based probabilistic inference in fatigue crack damage prognostics, J. Probabilistic Engineering Mechanics 29: 157-166. https://doi.org/10.1016/j.probengmech.2011.11.006.

[12] Gelman, A., (1996). Inference and monitoring convergence. In: Gilks WR, Richardson S, Spiegelhalter DT, editors. Markov chain Monte Carlo in practice. Chapman \& Hall/CRC, Boca Raton, FL.

[13] Virkler, D., Hillberry, B., Goel, P., (1979). The Statistical Nature of Fatigue Crack Propagation. J. Engineering Materials and Technology 101(2): 148-153. https://doi.org/10.1115/1.3443666.

[14] Moan, T., Ayala-Uraga, E., (2008). Reliability-based assessment of deteriorating ship structures operating in multiple sea loading climates, J. Reliability Engineering and System Safety 93(3): 433-446. https://doi.org/10.1016/j.ress.2006.12.008.

[15] Cremona, C., (1996). Reliability updating of welded joints damaged by fatigue, International Journal Fatigue 18(8): 567-575. https://doi.org/10.1016/S0142-1123(96)00011-4.

[16] DNV (2014). Fatigue assessment of ship structures. Classification Notes, No. 30.7. 
[17] Mansour, A., Wirsching, P., White, G., Ayyub, B., (1996). Probability based ship design: implementation of design guidelines: Ship Structure Committee, Report No. SSC-392.

Submitted: 12.05.2017. Mohammad Reza Zareei, PhD student, mrzarei@aut.ac.ir Department of Maritime Technology, Amirkabir University of

Accepted: $\quad$ 19.01.2018. Technology, 424 Hafez Avenue, Tehran 15916-34311, Iran. Mehdi Iranmanesh, Associated professor, corresponding author, imehdi@aut.ac.ir Department of Maritime Technology, Amirkabir University of Technology, 424 Hafez Avenue, Tehran 15916-34311, Iran. Tel: +98-21-64543119 Fax: +98-21-66412495 